\title{
Five years REACH - lessons learned and first experiences. I. an authorities' view
}

\author{
Christoph Schulte $^{1 *}$, Lars Tietjen ${ }^{1}$, Ann Bambauer ${ }^{2}$ and Andreas Fleischer ${ }^{2}$
}

\begin{abstract}
Background: Five years after entering into force, all the instruments of the REACH Regulation are active.

Results and conclusions: The first five years of REACH are characterised by a good cooperation between the European Chemicals Agency ECHA and the Member States authorities. As expected, much information for the most important chemicals on the European market was made available by the responsible registrant. However, the access to information for the public, researchers and downstream users needs to be expanded.

Most of the registrations submitted to ECHA didn't fulfil the expectations of the authorities with regard to transparency and quality. To assure a safe use of chemicals, manufacturers, importers and downstream users need to take over the responsibility for compliance and quality of the registration dossiers and the assessments including risk management. For the upcoming second registration deadline, registrants are requested to submit fully compliant dossiers. For many dossiers already submitted updating is required.

By September 2012, 84 Substances of very high concern are included in the candidate list. For 54 substances, proposals are submitted by the Member States and ECHA. Hence, the objective of the European Commission, to have 136 substances of very high concern included in the candidate list by end 2012 might be reached.

With regard to regulatory activities, identifying of at least PBT and $\mathrm{VPVB}$ substances is challenging due to missing information. In addition to that, the interface between restrictions and authorisations needs to be improved to include SVHC in (imported) articles in the authorisation regime.

The establishment of a national German REACH-CLP-Helpdesk was a well accepted support for the involved companies and played an important role in the communication between the concerned parties in order to implement the new elements of the regulations.
\end{abstract}

Keywords: REACH, Chemicals Regulation, SVHC, Authorisation, Candidate List, Restriction

\section{Background}

In June 2007, the European Chemicals Regulation $\mathrm{REACH}$ [1] entered into force, and the European Chemicals Agency ECHA was established in Helsinki. The period between the European Commission's presentation of the white paper "Strategy for a future Chemicals Policy" in 2001 [2] and the entry into force of REACH was characterised by hard negotiations between all parties and stakeholders involved. At least REACH was agreed as a good compromise.

REACH introduced an important change of paradigm into European chemicals legislation. Decades before

\footnotetext{
* Correspondence: Christoph.Schulte@uba.de

${ }^{1}$ Federal Environment Agency, Wörlitzer Platz 1, 06844, Dessau-Roßlau, Germany

Full list of author information is available at the end of the article
}

REACH it was the responsibility of the authorities of the Member States to evaluate the risks arising from manufacture, import and use of chemicals, e.g. as requested by the Existing Substances Regulation [3]. Member States authorities were also in charge of controlling the risks by risk legal management measures. One important instrument was the Chemicals Restrictions Directive [4].

One of the most important principles introduced by $\mathrm{REACH}$ is that it is for manufacturers, importers and downstream users to ensure that manufacturing, placing on the market or use of chemicals does not adversely affect human health or the environment (Art. 1(3)). Companies need to register the chemicals they manufacture or import with a yearly volume exceeding 1 ton. In addition to that the safe use for the whole life-cycle of a substance needs to be demonstrated in a chemical safety

\section{实 Springer}


report (CSR) as important part of the registration for substances with a yearly volume exceeding 10 tons. If risks are identified in the CSR, the responsible registrants need to propose and apply appropriate measures to adequately control the risks. These measures also need to be recommended to downstream users in the (extended) safety data sheet (Art. 14 (6)). The safety data sheet is also the instrument to communicate registered uses, hazards, risks and appropriate risk management measures within the supply chain.

However, the shift of responsibility is not complete. The Regulation also provides instruments for the authorities to control the risks of chemicals for workers, consumers and the environment. These instruments need to be considered when the measures proposed by the responsible registrants are not appropriate to guarantee a safe use for the whole life cycle of a chemical. Responsible for risk management using these instruments are still the authorities - the European Commission, the European Chemicals Agency, and the Member State Competent Authorities.

A well established instrument to regulate the risks of certain dangerous substances and mixtures are restrictions on manufacturing, placing on the market and use. New restrictions are introduced in Annex XVII of REACH. With the entering into force, existing restrictions already in place by the Chemicals Restrictions Directive [4] were directly integrated in Annex XVII.

Prerequisite to introduce a restriction is an unacceptable risk to human health or the environment which needs to be addressed on a Community-wide basis. The risk needs to be clearly described. Restrictions can be used in a very general way, and also targeted to address specific risks. Aim of a restriction is the adequate control of the risk to human health or the environment arising from the substance itself, in mixtures or in articles. It is also appropriate to introduce certain limits for residues, e.g. in articles.

A restriction can be suggested by a Member State or ECHA on request of the European Commission using the Annex XV-format. The proposal should also consider an estimation of the socio-economic effects of the restriction. The conformity with the requirements of this Annex needs to be checked by ECHA's Risk Assessment Committee (RAC), and the Committee on SocioEconomic Analysis (SEAC). Before ECHA forwards the restriction proposal to the European Commission to decide on, it is open for public comments. In addition, RAC and SEAC are requested to comment whether the suggested measures are appropriate to reduce the risks, and on the socio-economic impact. If the restriction proposal is considered as justified, and the risk needs to be addressed on a Community-wide basis, the European
Commission starts the regulatory procedure with scrutiny.

For consumer uses of CMR-substances (category 1A or $1 \mathrm{~B}$ ), also the European Commission can propose a restriction (Art. 68 (2)). For this type of proposal neither a formal public commenting period nor the Committees' opinions are needed for vote in the European Commission's REACH Committee of the Member States.

Besides the established instrument of restrictions, $\mathrm{REACH}$ introduced new instruments. The most important are the authorisation procedure for substances of very high concern (SVHC) and the candidate list. An important target of REACH is mentioned in Art. 55 "assuring that the risks from substances of very high concern are properly controlled and that these substances are progressively replaced by suitable alternative substances or technologies where these are economically and technically viable." In principle, this target will be achieved by introducing the process of authorisation for substances of very high concern.

$\mathrm{REACH}$ introduced the first time an authorisation requirement for common chemicals into the chemicals legislation of the EU. Authorisation is intended as an instrument to appropriately control the risks arising from manufacturing and use of SVHC. The properties defining a SVHC are described in Art. 57.

According to this Article the following properties are characteristic for substances of very high concern: carcinogenic, mutagenic or toxic for reproduction (CMR substances) - all of them due to category $1 \mathrm{~A}$ or $1 \mathrm{~B}$ in accordance with section 3.5-3.7 of Annex I to CLPRegulation [5]. With regard to the environment, substances of very high concern are characterized by persistent, bioaccumulative and toxic properties (PBTsubstances), or very persistent and very bioaccumulative properties (vPvB-substances). The criteria for PBT and $\mathrm{vPvB}$ are set out in Annex XIII of REACH.

In addition, substances need to be considered as being of very high concern if there is scientific evidence of probable serious effects to human health or the environment which give rise to an equivalent level of concern to that of CMR-, PBT-, or vPvB-substances. Art. $57 \mathrm{f}$ ) is suggesting as substances of an equivalent concern such as those having endocrine disrupting properties or those having persistent, bioaccumulative and toxic properties or very persistent and very bioaccumulative properties, which do not fulfil the criteria of Annex XIII.

A SVHC will become subject to authorisation by inclusion into Annex XIV of the Regulation. Responsible for the decision on inclusion in Annex XIV is the $\mathrm{REACH}$ committee of the European Commission. In advance of this decision, ECHA is requested to recommend which substances of the candidate list should be included in Annex XIV with high priority. Once 
included in Annex XIV, production and use of the substance in the EU is limited by a sunset date. From this date on, every single use needs to be assessed by ECHA and authorised by the European Commission for a limited period on request of manufactures or users.

Prior to inclusion into Annex XIV, an agreement must be reached between the Member States to consider a chemical as a SVHC. As a first step, it needs to be demonstrated that the criteria of Art. 57 are fulfilled. The proposal for a SVHC and the comparison with the criteria needs to be documented in an Annex XVdossier.

Twice a year dossiers suggesting a chemical as SVHC could be submitted to ECHA. Following a public commenting period, the proposal is forwarded to the Member State Committee (MSC). Once the MSC agrees unanimously, the SVHC is taken up in the candidate list for eventual inclusion in Annex XIV (Art. 58 (1)) - the candidate list. If the MSC does not agree unanimously, the proposal is forwarded to the European Commission for decision.

To verify the quality and compliance of registrations, $\mathrm{REACH}$ introduced the instrument of dossier evaluation. Dossier evaluation is an important task of ECHA. One element of dossier evaluation is the examination of testing proposals (TPE) received with the registration dossiers. A dossier containing a testing proposal is automatically considered for dossier evaluation. The other element of the dossier evaluation is the compliance check with the possible outcomes: no administrative action needed, decisions, draft decisions for comments, and quality observation letters to inform the registrant about deficiencies of the dossier and to consider updating of the registration.

The other instrument of evaluation is substance evaluation, which is due to the Member States authorities. ECHA's role is to coordinate the activities, e.g. by compiling the Community Rolling Action Plan (CoRAP). Substance evaluation started in 2012.

In the context of REACH and the CLP Regulation [5] for the first time in EU chemicals' regulation the Member States have been requested to establish national helpdesks to provide advice to the various stakeholders. Due to the fact that many newcomers are affected by their new responsibilities under both regulations the main target groups are medium and small enterprises.

The German REACH-CLP-Helpdesk is located at the Federal Institute for Occupational Safety and Health (BAuA) as the assigned competent authority. It is established as a network of experts from BAuA, the Federal Institute for Risk Assessment (BfR), the Federal Environmental Agency (UBA) and the Federal Institute for Materials Research and Testing (BAM) [6]. It provides information and guidance in relation to the implementation of REACH and the CLP-Regulation. In particular support is offered regarding the registration, evaluation and authorisation procedures as well as the classification and labelling of chemicals. Building on ECHA's Guidance documents short understandable guidance brochures were developed in German language in order to allow small and medium enterprises an easier access to the different operational steps of implementation. Stakeholders are supported by the information provided on the website, including summaries of topics related to the different operational phases. The Helpdesk is organising conferences and workshops. In addition, the helpdesk can be approached with specific questions and requests for discussions on substance and company related concerns.

\section{Results and Discussion}

November 2010 - a huge amount of information became available

By the first registration deadline in November 2010, nearly 4,300 substances were registered in approximately 25,000 technical dossiers. After five years REACH, the responsible manufacturers and importers submitted 28,669 registrations for 5,894 substances (16.08.2012) [7]. Hence, for all substances with a manufacturing or import volume of more than 1,000 tons/year and manufacturer/importer, information on substance identity, additives, impurities and constituents, manufacturing and use within the complete supply chain, data on physico-chemical properties, hazards and risks should be available. It is also requested that the submitted data set includes information on fate and behaviour in the environment, studies on toxic and ecotoxic effects, and the proposed risk management measures.

The registrations also comprise other than high production volume substances: 1 . substances that are carcinogenic, mutagenic or toxic for reproduction for which placing on the market within the EU is intended to be continued, and 2. substances classified as toxic for the environment R50/R53 if they are manufactured or imported with more than 100 tons per year and manufacturer or importer.

The registration data for these chemicals are stored in ECHA's databases, and are mainly available to the competent authorities of the Member States. Many data are also publicly available, e.g. via ECHAs homepage [7] or the eChemPortal of the OECD [8]. Additionally, the access to testing protocols and results performed by manufacturers and importers needs to be extended for research issues in order to achieve a comparison with results of independent research. Open discussion is expected to increase the knowledge about chemicals and their interactions with humans and the environment. 


\section{Many registrations show deficiencies}

According to ECHA [9] and also due to the experiences of the German authorities a significant part of the registrations has deficiencies. One example is the registration as intermediate. In $2011 \mathrm{ECHA}$ reported that $86 \%$ of the screened 400 registrations for intermediates did not contain sufficient information to demonstrate that the conditions for a registration as intermediate are fulfilled [9].

Another example is a screening of certain UVCB (Unknown or Variable composition, Complex reaction products or Biological materials) substances of the petrochemical- and the coal industry performed by the German Federal Environment Agency. These substances are expected to contain certain poly aromatic hydrocarbons (PAH) known for their CMR and PBT properties. However, the registrants did not identify the PBT constituents in the registration dossiers screened. In addition, anthracene and derivatives, which are already included in the candidate list as SVHC were not considered in the dossiers though these substances are expected to be constituents of the screened UVCBs. Additionally, registrations for UVCBs often show deficiencies with respect to substance identity [9].

Other deficiencies, e.g. observed while screening for potential PBT and $\mathrm{vPvB}$ substances are missing testing proposals, misuse of waiving, and missing documentation of structure activity relationships used to fulfil certain data requirements. In addition, exposure assessment, especially refinement and on-site risk management are used in an intransparent manner without documentation.

\section{Substances of very high concern and authorisations}

The SVHC included in the candidate list for eventual inclusion in Annex XIV were mostly CMR substances which were already classified and labelled as such see Figure 1. Six of them were additionally identified as PBT and $\mathrm{vPvB}$ (anthracene oil and derivates). Three PBT-substances were included in the candidate list with regard only to environmental concerns only, one PBT which additionally fulfils the criteria for vPvB (short chain chlorinated paraffins), one vPvB (musk xylene), and one endocrine disrupting substance fulfilling the criteria of Art. $57 \mathrm{f}$ ) - equivalent level of concern (4-tert-octylphenol).

Following a visit of ECHA in March 2010, the EUCommissioners of the General Directorates Enterprise, Antonio Tajani, and Environment, Janez Potočnik, jointly agreed to strengthen the status of the candidate list [10] and increase substitution of SVHC. They defined as a target to include 136 SVHC in the candidate list by end of 2012. In December 2011, the Commission recalled this objective [11]. By September 2012, 84 substances were included in the list. In addition to that, 54 proposals for SVHC were submitted by the Member States and ECHA in September 2012 [12].

Till September 2012, only 10 Member States and Norway submitted proposals for SVHC. In total 145 dossiers were submitted, seven were withdrawn by the submitting authorities before voting in the Member State Committee. ECHA submitted 55 dossiers, France 17, and Germany 32. For more details see Figure 2.

\section{Experiences identifying SVHC / PBT-substances}

Under the old legislation, a PBT-expert group was established as a subgroup of the Technical Committee on New and Existing Substances - TCNES. The group prioritised 127 existing substances for an in-depth evaluation. 27 PBT and vPvB-substances were identified in 12 meetings in six years [13]. The work of the group was finalised with the last meeting in March 2008. Under $\mathrm{REACH}$, the German authorities developed a strategy how to proceed with the PBT- assessment and how to identify PBT and vPvB-candidates. The first step of the strategy was to transfer the results of the PBT-subgroup into the legislative context of REACH.

In the PBT-subgroup, the German experts were rapporteur for anthracene and six anthracene derivatives. In order to transfer the results of this evaluation into the legislative framework of $\mathrm{REACH}$, Germany suggested these substances as SVHC due to the persistent, bioaccumulative and toxic properties. The Member State Committee (MSC) unanimously supported these proposals. In October 2008 and January 2010 anthracen and six anthracen derivatives were taken up in the candidate list for eventual inclusion in Annex XIV (Art. 58 (1)).

Although the criteria of Annex XIII were adapted to the scientific progress with regard to PBT and $\mathrm{vPvB}$ properties in March 2011 [14], no decision concerning a PBT or vPvB substance was made in 2011 and 2012. No proposal for a PBT-substance was submitted between August 2009 and July 2012.

The PBT-subgroup also identified 1,2,3-trichlorobenzene and 1,2,4-trichlorobenzene as PBT due to the criteria of the TGD [15]. Hence, Germany suggested both and additionally 1,2,5-trichlorobenzene as SVHC, and for inclusion in the candidate list. The dossiers were submitted in August 2010. However, the proposals were not supported by the experts of other Member States, and hence Germany withdrew the proposals following an intensive discussion in the Member State Committee.

This case, and the lack of proposals for additional PBT or vPvB substances as SVHC made clear the need to reestablish a technical discussion about PBT-issues. Hence, the German Federal Environment Agency organised a PBT-workshop between Member States experts and ECHA in Dessau in November 2010, followed by several 


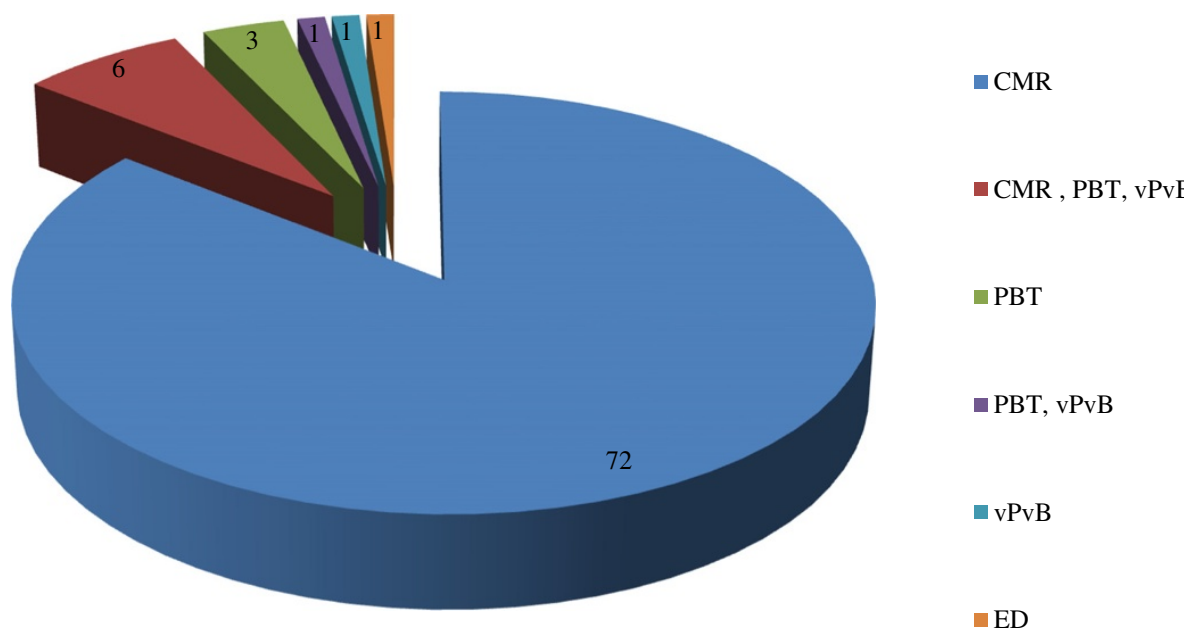

Figure 1 Substances of very high concern agreed by the Member State Committee and included in the candidate list for eventual inclusion in Annex XIV.

expert meetings to co-ordinate the activities on identification of additional PBT and $\mathrm{vPvB}$-candidates. The activities ended up in the establishment of a PBT expert group by ECHA mandated by the meeting of the Member States competent authorities.

The second step of the German PBT-strategy was to identify and to prioritise additional potential PBT- and $\mathrm{vPvB}$-candidates among the substances pre-registered for 2010. During this prioritisation process the Federal Environment Agency made another experience: According to Annex IX of the Regulation, a standard requirement is information on biological degradation (Annex IX, 9.2.1), especially tests simulating the degradability in different environmental compartments (simulation studies). Although most of the substances were registered with a production or import volume exceeding the 100 ton/year level, and also the 1,000 ton/year level, no data on degradability and degradation half lives was included in the dossiers with the exception of ready biodegradation tests.

\section{Art. 57 f) endocrine disruptors}

In the EU an intensive discussion on endocrine disrupting chemicals is ongoing. The criteria to assess this mechanism of action and the assessment of effects as adverse are currently under development. However, certain circumstances are already clarified, and also a conceptual framework for the testing and assessment of endocrine disrupting chemicals was developed by the OECD [16]. Using this framework, and all data available for this substance, 4-tert-octylphenol was suggested as the first case for which an equivalent level of concern according to Art. $57 \mathrm{f}$ ) could be demonstrated due to the endocrine disrupting effects in the environment,

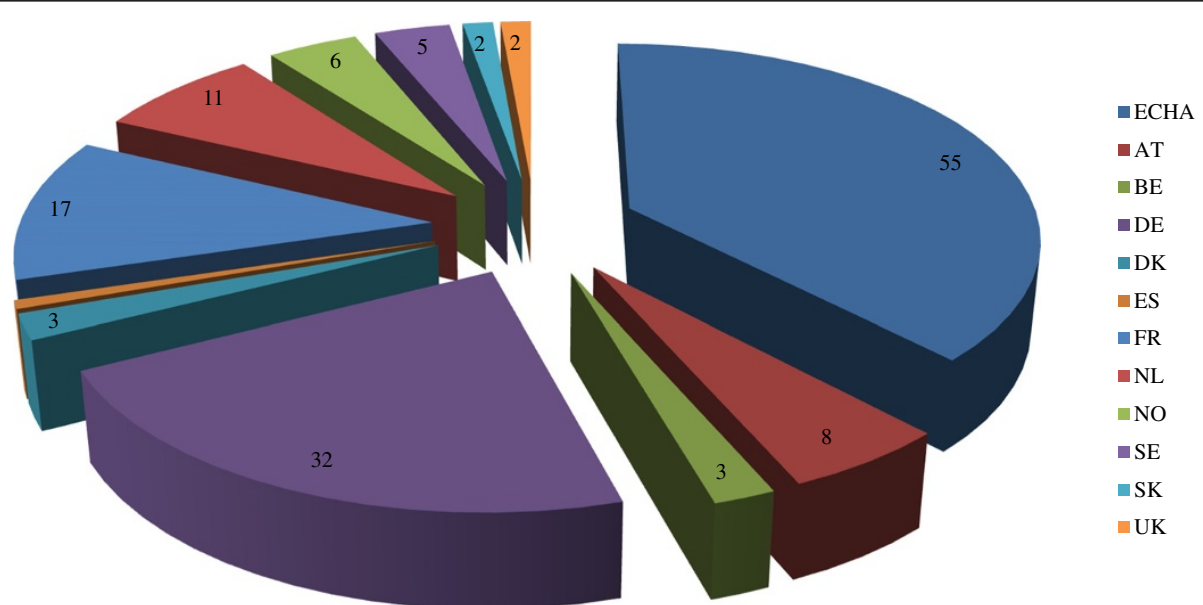

Figure 2 No of Annex XV dossiers prepared by ECHA and the Member States (including withdrawn dossiers). 
especially in fish populations. The proposal was agreed in the Member State Committee in autumn 2011, and 4tert-octylphenol was included in the candidate list as the first Art. 57 f)-case.

\section{Substances in substances}

When preparing the Annex XV-Dossiers for anthracene and anthracene derivatives it became clear that environmental exposure to these substances is mainly due to constituents in other UVCB-substances of different sources. Like other PAH of concern, anthracene and anthracene derivatives are not registered, because these substances are neither produced nor marketed as single substances. The environmental exposure is due to UVCB-constituents. Hence regulating PAH as certain single substances is not appropriate to address the concerns. Therefore the concept of substance in substances (SiS) was developed.

The intention of the SiS concept is to regulate substances of very high concern as constituents or impurities in substances, mixtures, and in articles. In principle this is already realised in certain entries of Annex XVII. As a first case, Germany is preparing an Annex XV dossier for the PAH benzo[a]pyrene as substance in substance. Benzo[a]pyrene is the most prominent PAH known for CMR and vPvB-properties, and already regulated in mixtures for consumer uses (Annex XVIII), as an impurity in toys and as extender oil for the production of tyres or parts of tyres (Annex XVII, entry 50).

\section{Authorisations}

By September 2012, 14 SVHC are already included in Annex XIV. Among these substances are four phthalates and some lead pigments. For 11 of these substances, the last possibility to apply for an authorisation is in 2013 [17]. If no authorisations are applied for and granted, marketing and use of these SVHC will terminate between August 2014 and August 2015. In addition, ECHA suggested 10 substances to be prioritised for inclusion into Annex XIV in 2012.

\section{Restrictions}

By end of June 2012, 14 proposals for restrictions were submitted. Examples are a restriction for lead and its compounds in jewellery, certain phenylmercury derivatives, and a restriction proposal for four phthalates which are already identified as SVHC and included in Annex XIV, the authorisation list. Additional restrictions for some nonylphenols and nonylphenol ethoxylates were submitted in August 2012.

\section{Interfaces authorisation - restrictions}

ECHA and Member States authorities learned an important lesson while proposing SVHC for inclusion in the candidate list, and in Annex XIV for authorisation. The experience revealed that authorisation is not always the appropriate measure to reduce and to control the risks of SVHC. Authorisation is focusing on the use of a substance and on placing on the market for a certain use. Especially for SVHC released into the environment as constituents or residues in articles, authorisation is not helpful to reduce emissions, because articles are not subject to authorisation. In addition, authorisation is not appropriate to introduce maximum residue levels for substances of very high concern in articles. The need to minimise the levels of SVHC in articles might especially be considered for imported articles. Hence, for certain SVHC, a restriction might be a better alternative to control the risks adequately.

In order to describe the best option to control the risks of a substance, ECHA and the Member States competent authorities invented a new instrument not foreseen in the REACH-Regulation: The analysis of risk management options (RMO-analysis). The RMOanalysis is distributed to ECHA, the European Commission, and the Member States in advance of announcing the intended option in the Registry of intention. The RMO-analysis is subject to discussions and comments in the risk management expert meetings (RiME), which are organised three times a year by Member States authorities with financial and organisational support by ECHA.

\section{Dossier evaluation}

In the period between 01.06.2008 and 31.07.2012 ECHA received 563 dossiers with testing proposals [18]. 439 of the testing proposals included proposals for testing vertebrate animals. To assure that the German standards for assessing chemicals risk are completely transfered in the community procedures, the German authorities are aiming at taking an active part and commenting on the testing proposal examination (TPE), and the other elements of dossier evaluation.

According to REACH, Art. 41(5), the number of dossiers selected for compliance check shall be at least $5 \%$ of the total received by the Agency for each tonnage band. Excluding the registrations for on-site intermediates, 19,772 dossiers were submitted to ECHA by the first registration deadline [18]. To fulfil the $5 \%$ requirement, 989 dossiers need to be checked for compliance. ECHA is intending to fulfil this requirement till the next registration deadline by December 2013. The outcome of the 418 compliance checks by end of May 2012 is shown in Figure 3. 


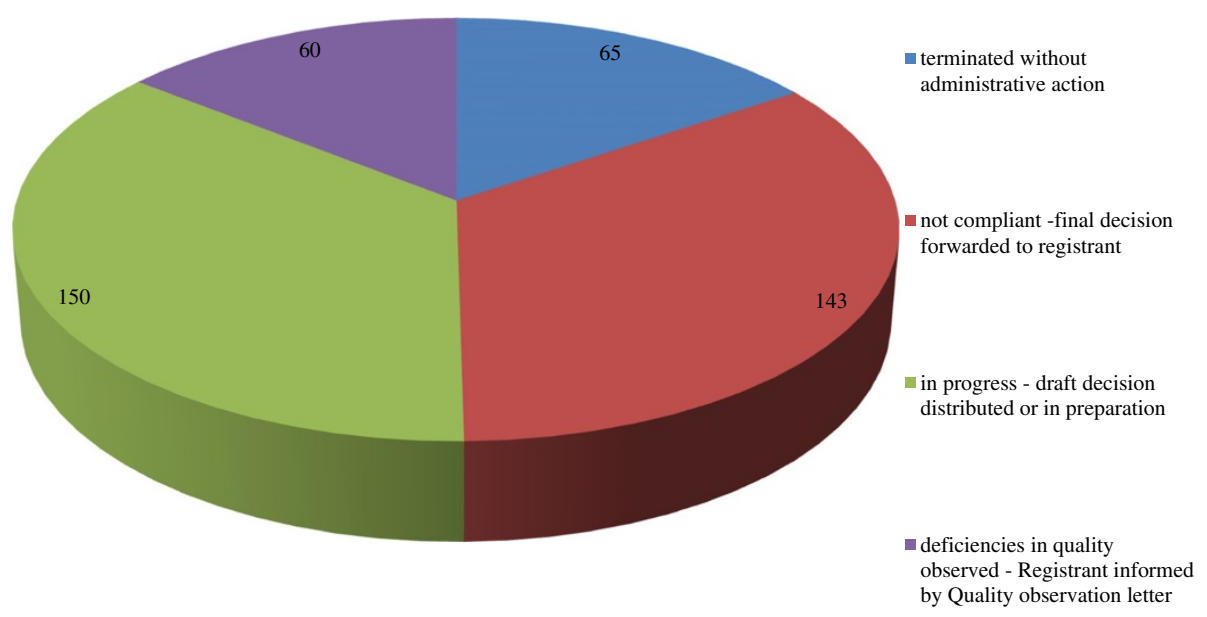

Figure 3 Outcome of 418 compliance checks from 01.06.2008 - 31.05.2012. Source: ECHA Newsletter 3/2012.

\section{Substance evaluation and the community rolling action plan}

The Community Rolling Action Plan (CoRAP) specifies the substances that Member States assured to evaluate over a period of three years. The first CoRAP includes 90 substances nominated by 17 Member States for substance evaluation. In 2012, 36 substances are evaluated. The results must be reported to ECHA within 12 months following the publication of the CoRAP, for 2012 at the latest at 2013, February $28^{\text {th }}$. Germany decided to evaluate five substances in 2012:

\section{4,4'-isopropylidenediphenol (Bisphenol A) is a} suspected Endocrine Disruptor. The substance is mainly used for the production of polycarbonate with a very high production amount of approximately 1 Mio. tons per year. It is also used for certain consumer products, and regularly measured in the environment.

2. N-1-naphthylaniline is identified as a potential PBTsubstance by the screening procedure of the Federal Environment Agency and prioritised for further assessment due to the wide dispersive use.

Unfortunately the information submitted with the registration dossier does not allow a conclusion whether the PBT-criteria are fulfilled or not.

3. N-hexane was selected for substance evaluation due to the CMR-properties and neurotoxic effects. There are several registrations of different registrants leading to a higher tonnage and exposure level than concluded in the single dossiers. The intention of the substance evaluation is to evaluate the risks arising from the aggregated exposure.

4. 2,2'-Iminodiethanol is also registered by several companies suggesting a higher exposure level than estimated in the single dossiers. The substance is suspected to be transformed in the body to CMR substances.

5. Polyhaloalkene (HFO $1234 \mathrm{yf}$ ). Due to the lower global warming potential this substance is intended to be used world-wide in air conditions especially in cars as substitute for $\mathrm{R} 134 \mathrm{a}$. The estimated use volume is extremely high leading to a high environmental exposure. The substance evaluation is aiming at assessing the risks arising from high production and use levels including environmental degradation products.

\section{Information on SVHC in articles}

Another important experience concerns the duty to communicate information on substances in articles, especially on SHVC. According to Art. 33(1), the supplier of an article shall provide the recipient of the article with sufficient information to allow safe use of the article. A prerequisite is a concentration of the SVHC in the article above $0.1 \%$ weight by weight $(\mathrm{w} / \mathrm{w})$. The minimum information requirement is the name of that SVHC. On request, this information also needs to be provided to consumers, free of charge, within 45 days of receipt of the request.

The first experiences with regard to these duties indicate that consumers assess the possibilities to address requests to retailers as too formal and laborious.

As part of a research project supported by the Federal Environment Agency an electronic tool has been developed using the barcode of a product to generate a request via email. This request is directly forwarded to the responsible producer or importer of the product. Currently, for every request the contact data of the requesting person need to be entered in the electronic request tool, because an individual response has to be sent to the requesting person. The electronic tool is available via internet (e.g. [19]). As a next step it is intended to generate the request automatically using a personal smartphone by scanning 
the barcode of the article. This seems to be a promising way forward to involve the public, and retail in the SVHC in articles issue.

\section{The German REACH-CLP-Helpdesk}

The German REACH-CLP helpdesk plays an important role when companies get involved with the new regulations. This becomes apparent from the strong demand for information which was noted during the last five years. The helpdesk provides information on demand by answering in total 15.300 enquiries and arranging 114 bilateral talks to industry. In the year 2008 companies had to fulfil their duty to pre-register phase-in substances in order to take advantage from the transitional regime providing registration deadlines depending on the tonnage band. Looking back on a continuous increase of enquiries in the beginning of 2008 the maximum peak was reached with 1100 enquires in November close to the deadline of 1 December 2008.

Since then, a steady number of requests around 200 per months kept the helpdesk busy showing the need of companies to get specific answers to their company related concerns. The next phase of $\mathrm{REACH}$, the first registration deadline was reached on 1 December 2010, accompanied by an increase of enquiries up to 2800 during this year (Figure 4).

The information provided by the helpdesk was extended in connection to the actual operational sequences of the regulation. Besides the general information and guidance, up to today 340 answers to frequently asked questions (FAQ) are available on the helpdesk website [6]. The increasing interest in information is reflected by 6.4 million page views on the website in 2011. Since 200718 information events were organised by the helpdesk with a total of 3200 participants. Feedback to the helpdesk shows the great interest of enterprises in receiving brief understandable guidance (documents) inter alia for registration, communication in the supply chain and legal requirements with respect to the identification of SVHC or substances to be included in Annex XIV.

The topics of enquiries answered by the helpdesk changed during the different operational phases of REACH or when new regulation entered into force: In the beginning pre-registration and registration issues were on the agenda, later on enterprises were increasingly interested in classification and labelling. Questions related to the obligations of downstream users and the changes in the requirements for safety data sheets were raised from the beginning up to today.

In conclusion the establishment of a national helpdesk was a well accepted support for the involved enterprises. In particular subject and company tailored answers, talks and guidance documents were requested very often. Although many new requirements are introduced by $\mathrm{REACH}$, at the end successful registrations were achieved and the commitment and the resources put into the helpdesk paid off.

\section{Conclusions}

The first five years of REACH were characterised by a good cooperation between ECHA and the national authorities responsible for chemicals legislation. As expected much information for the most important

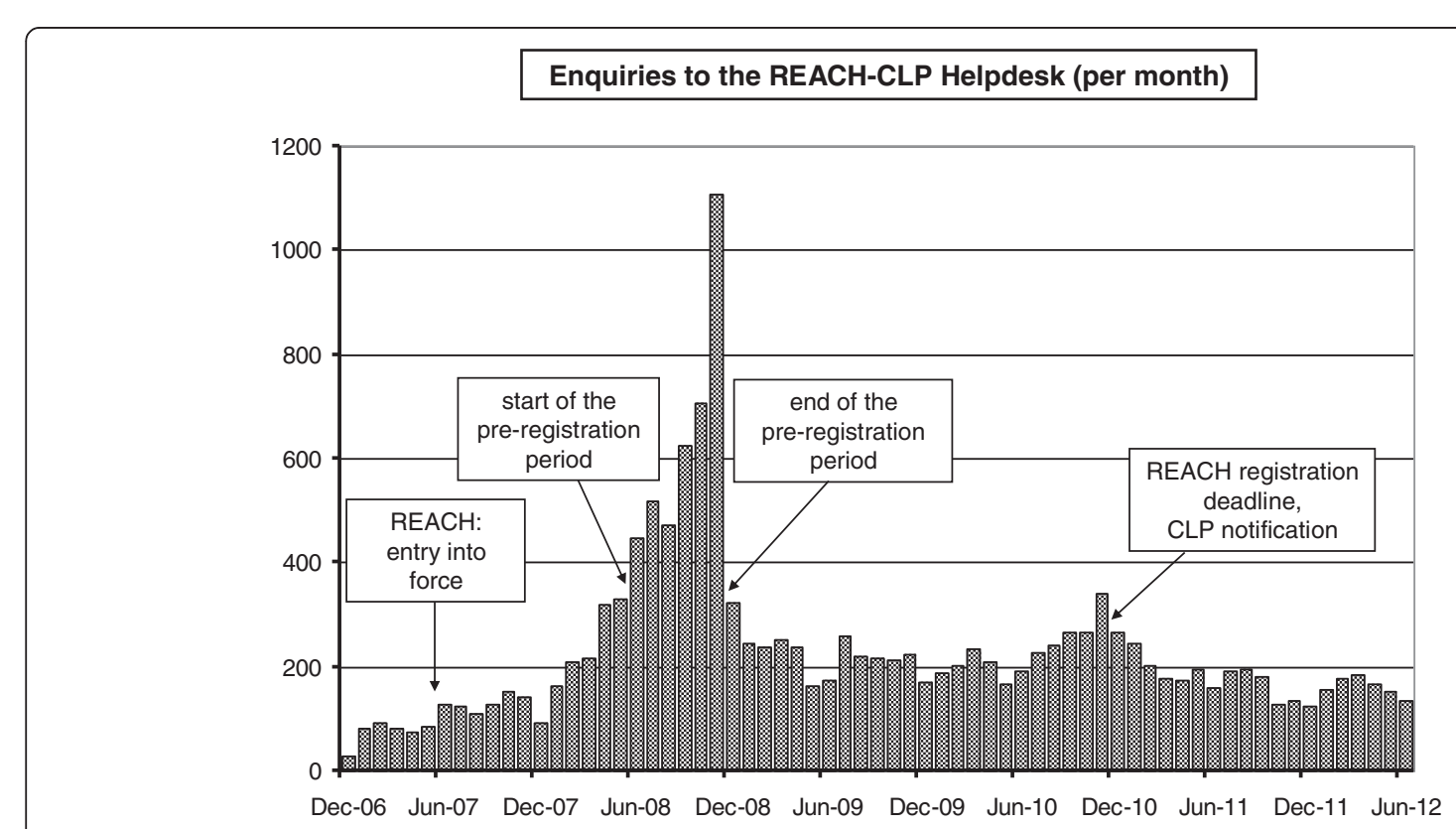

Figure 4 Overview of the enquiries of companies to the REACH-CLP-Helpdesk, December 2006 - June 2012. 
chemicals on the European market became available to the authorities. Many data are also publicly available, e.g. via ECHAs homepage or the eChemPortal of the OECD. To challenge the results of testing performed by manufacturers and importers of the substances, access to testing protocols and results needs to be extended. Comparison with results of independent research and open discussion of the results will increase the knowledge about chemicals and their interaction with humans and the environment.

The establishment of a REACH helpdesk was well accepted by the involved stakeholders. The steady demand for information showed the need for a national German system of support to assist in particular medium and small enterprises. Regarding the continuous requests for information and advice the expectations set in the helpdesk have been fulfilled, the helpdesk can be recommended as a supporting system for the next registration phase in 2013.

Nevertheless, communication in the supply chain needs to be improved. Downstream users must be better integrated, and also suppliers and consumers need to be informed, especially about substances of very high concern in articles.

The change of paradigm - the transfer of the responsibility to assess the risks from Member State authorities to manufacturers, importers, and downstream users is not completely fulfilled yet. Registrants are requested to take over their responsibility for compliance and quality of the registration dossiers and the assessments. Manufacturers, importers, and downstream users need to be aware the duty to ensure that manufacturing, placing on the market or use of chemicals does not adversely affect human health or the environment. A prerequisite is a complete and compliant assessment of the risk including the transparent documentation of risk management.

With regard to risk management, registrants are in a position to focus a registration on certain important and well controlled uses, e.g. by considering other uses as "uses advised against". This might be an option for registrants to fulfil the responsibility for the safety of a manufactured or imported substance which may decrease the priority for authorities to regulate the respective substance.

While trying to identify substances of very high concern for regulatory action, the German Federal Environment Agency learned that especially for potential PBT and vPvB-substances the data submitted did not allow a direct comparison with the numeric criteria of Annex XIII in most cases. The dossiers checked are often not complete. Sometimes waiving was misused, or QSAR models were used unjustified and without the necessarily documentation. Therefore the target for the registrations announced for 2013 is a compliant set of information, and a well-documented, transparent risk assessment. For substances already registered, updating of the registration is a suggested way forward to fulfil the expectations of the authorities and the public. As an ultimate option for non-compliant registrations it is suggested to introduce a possibility for ECHA to withdraw a registration.

With regard to PBT and $\mathrm{vPvB}$ substances, we would suggest to extend the responsibility of the registrants to include a part of the SVHC-identification. If the results of screening for persistency and bioaccumulation indicate a substance is a PBT or $\mathrm{vPvB}$ candidate, it should be the task of the registrant to evaluate whether the criteria of Annex XIII are fulfilled or not.

As a last important aspect it is suggested to improve the interface between restriction and authorisation. Especially the gap of SVHC in imported articles under the authorisation regime needs to be closed.

\section{Methods}

Literature review and analysis of data obtained in the review were performed to achieve the aim of the study.

\begin{abstract}
Abbreviations
CLP: Classification labelling, packaging; CoRAP: Community Rolling Action Plan; ECHA: European Chemicals Agency; EU: European Union; MSC: Member State Committee; PAH: Poly aromatic hydrocarbons; PBT: Persistent bioaccumulative and toxic; POP: Persistent organic pollutant;

QSAR: Quantitative structure activity relationship; RAC: Risk Assessment Committee; REACH: European Chemicals Regulation EC No. 1907/2006 on Registration Evaluation, Authorization and Restrictions of Chemicals; RiME: Risk Management Expert; RMO: Risk Management Option; SEA: Socioeconomic Analysis; SVHC: Substance of Very High Concern; TPE: Testing proposals examination; UVCB: Unknown or Variable composition complex reaction products or biological materials; $\vee P \vee B$ : very persistent very bioaccumulative.
\end{abstract}

\section{Competing interests}

The authors declare that they have no competing interests.

\section{Authors' contributions}

All authors contributed in equal parts to this publication. All authors read and approved the final manuscript.

\section{Author details}

${ }^{1}$ Federal Environment Agency, Wörlitzer Platz 1, 06844, Dessau-Roßlau, Germany. ${ }^{2}$ Federal Institute for Occupational Safety and Health, Friedrich-Henkel-Weg 1-25, 44149, Dortmund, Germany.

Received: 20 September 2012 Accepted: 30 September 2012 Published: 20 October 2012

\section{References}

1. Regulation (EC) No 1907/2006 of the European Parliament and of the Council of 18 December 2006 concerning the Registration, Evaluation, Authorisation and Restriction of Chemicals (REACH), establishing a European Chemicals Agency, amending Directive 1999/45/EC and repealing Council Regulation (EEC) No 793/93 and Commission Regulation (EC) No 1488/94 as well as Council Directive 76/769/EEC and Commission Directives 91/155/EEC, 93/67/EEC, 93/105/EC and 2000/21/ EC. Official Journal of the EU 2006, L396:1-849.

2. Commission of the European Communities: White paper - Strategy for a future Chemicals Policy. COM(2001) 88 final 2001, :32p. 
3. Council Regulation (EEC) No $793 / 93$ of 23 March 1993 on the evaluation and control of the risks of existing substances. Official Journal of the European Communities 1993, L 84/:1-75.

4. Council Directive of 27 July 1976 on the approximation of the laws, regulations and administrative provisions of the Member States relating to restrictions on the marketing and use of certain dangerous substances and preparations (76/769/EEC). Official Journal of the European Communities 1976, L 262:201-203.

5. Regulation (EC) No 1272/2008 of the European Parliament and of the Council of 16 December 2008 on classification, labeling and packaging of substances and mixtures, amending and repealing Directives 67/548/ EEC and 1999/45/EC, and amending Regulation (EC) No 1907/2006. Official Journal of the EU 2008, L 353:1-1355.

6. BAUA: REACH-CLP-Helpdesk. http://www.reach-clp-helpdesk.de/.

7. European Chemicals Agency: Registered substances. http://echa.europa.eu/information-on-chemicals/registered-substances.

8. OECD: The Global Portal to Information on Chemical Substances. http://www.echemportal.org/.

9. European Chemicals Agency: Report on the Operation of REACH and CLP. Helsinki: 2011.

10. European Commission: Commission speeds up registration and authorisation for safer chemicals. Memo/10/102 (25.03.2010). http://europa.eu/rapid/pressReleasesAction.do?reference=MEMO/10/102.

11. Council of the European Union: REACH and Candidate List of Substances of Very High Concern - Information from the Commission; Council-Document 14488/11. 2011.

12. European Chemicals Agency: Registry of intentions. http://echa.europa.eu/web/quest/addressing-chemicals-of-concern/registryof-intentions.

13. EU, Joint Research Centre, Institute for Health and Consumer Protection: European Chemical Substances Information System (ESIS). http://esis.jrc.ec.europa.eu/index.php?PGM=pbt.

14. Commission Regulation (EU) No 253/2011 of 15 March 2011 amending Regulation (EC) No 1907/2006 of the European Parliament and of the Council on the Registration, Evaluation, Authorisation and Restriction of Chemicals (REACH) as regards Annex XIII. Official Journal of the EU 2011, L 69:7-12.

15. European Commission - Joint Research Centre: Technical Guidance Document in support of Commission Directive 93/67/EEC on risk assessment for new notified substances and Commission Regulation (EC) No 1488/94 on risk assessment for existing substances and Commission Directive (EC) 98/8 on biocides. 2nd Edition. Luxembourg: Luxembourg: European Commission; 163-164. Part II.

16. OECD: Conceptual Framework for the Testing and Assessment of Endocrine Disrupting Chemicals. http://www.oecd.org/document/58/0,3746, en_2649_34377_2348794_1_1_1_1,00.html.

17. European Chemicals Agency: Authorisation List. http://echa.europa.eu/addressing-chemicals-of-concern/authorisation/ recommendation-for-inclusion-in-the-authorisation-list/authorisation-list.

18. European Chemicals Agency: Evaluation statistics. ECHA Newsletter $2012,: 23-24$.

19. BUND - Friends of the Earth Germany: Gifttrage stellen! http://www.bund.net/themen_und_projekte/chemie/auskunft_fordern/ gifftrage_stellen

\section{Submit your manuscript to a SpringerOpen ${ }^{\circ}$ journal and benefit from:}

- Convenient online submission

- Rigorous peer review

- Immediate publication on acceptance

- Open access: articles freely available online

- High visibility within the field

- Retaining the copyright to your article

Submit your next manuscript at $\gg$ springeropen.com 